\title{
Un manuscrito complutense redivivo Ms. griego 442 = Villa-Amil 22
}

\author{
Natalio FERNÁNDEZ MARCOS * \\ Instituto de Filología - CSIC, Madrid
}

\section{INTRODUCCIÓN}

El manuscrito 442 del catálogo de Rahlfs, 22 del catálogo de VillaAmil, 116-Z-36 según la antigua signatura, se encuentra hoy bajo la sigla Biblia (en griego), s. XV, en la Biblioteca Histórica «Marqués de Valdecilla» de la Universidad Complutense de Madrid. Perteneció a los fondos del Colegio Mayor de San Ildefonso, fundado por el Cardenal Cisneros en Alcalá (= Complutum) entre 1499 y 1508 , fondos que fueron trasladados a Madrid en $1841^{1}$.

Los antiguos catálogos informan de que fue uno de los manuscritos utilizados para la edición de la columna griega de la Políglota de Alcalá, pero sólo los estudios de F. Delitzsch ratificaron esta información ${ }^{2}$. Se trata de una copia incompleta de un manuscrito de la Biblioteca Marciana de Venecia, enviada por el Senado de Venecia a Alcalá a petición del Cardenal Cisneros. Dicho manuscrito procedía

*natalio@filol.csic.es

1 A. RAHLFS, Verzeichnis der griechischen Handschriften des Alten Testaments (Berlín 1914) págs. 123-124; J. VIlla-Amil y CASTRO, Catálogo de los manuscritos existentes en la biblioteca del Noviciado de la Universidad Central (procedentes de la antigua de Alcalá). Parte I: Códices (Madrid 1878) págs. 5-6.

${ }^{2}$ F. DelitzsCH, Fortgesetzte Studien zur Entstehungsgeschichte der Complutensischen Polyglotte (Leipzig 1886) págs. 13-28.

Sefarad 65 (2005) págs. 65-83

(c) CSIC

ISSN 037-0894 
del legado del cardenal Besarión (1403-1472) a la biblioteca Marciana. Al parecer, es la copia a la que alude Cisneros en el Prólogo al Lector de la Políglota Complutense. En efecto, después de mencionar los manuscritos de la Biblioteca Vaticana prestados por el papa León $\mathrm{X}$, prosigue el cardenal: «Quibus etiam adiunximus alia non pauca: quorum partem ex Bessarionis castigatissimo codice summa diligentia transcriptum Illustris Venetorum Senatus ad nos misit». Es la única noticia con que contamos sobre esta copia realizada en Venecia. Delitzsch no encontró ningún documento que lo atestiguara en los archivos de la Biblioteca Marciana, pero sometió a un riguroso estudio los manuscritos procedentes de los fondos del cardenal Besarión, y llegó a la conclusión de que el manuscrito Graecus 5 de la Biblioteca Marciana (= 68 del catálogo de Rahlfs), una Biblia completa del s. XV, era el mejor candidato al que correspondía el adjetivo «castigatissimus» empleado por Cisneros. Esta sospecha se confirmó al constatar que la Complutense rellenaba con este manuscrito de Venecia las lagunas del ms. 330 de la Biblioteca Vaticana ${ }^{3}$. Sin embargo, seguimos sin saber la razón de por qué la copia fue realizada de forma incompleta, ya que en el manuscrito de Madrid faltan el Pentateuco, Josué, Salmos, Job, Sira y todos los Profetas.

Delitzsch, en el estudio antes citado, imprimió el canto de Débora (Jueces 5) y la elegía de David (2 Samuel 1,19-26) del manuscrito de Madrid, según una copia que le procuró el bibliotecario de la Complutense, Pascual de Gayangos. Todavía el 21 de julio de 1860 estuvo en Madrid el Dr. S. P. Tregelles, examinó el manuscrito y pudo constatar la extensión de la copia, la misma que señala Rahlfs en su catálogo, con una pequeña modificación: menciona el libro de Ester sin el añadido de Rahlfs «la partie qui existe dans l'hébreu» (la parte que existe en hebreo), nota que Rahlfs tomó probablemente de Ch. Graux y A. Martin, Notices sommaires des mss. Grecs d'Espagne et de Portugal (París 1892) págs. 125-139.

No volvemos a tener noticias de esta manuscrito de Madrid hasta que comienzan a publicarse las ediciones críticas de los libros históricos en la serie de Gotinga. W. Kappler, en su edición de 1 Maca-

${ }^{3}$ F. DelitzSCH, Fortgesetz.te, pág. 26. 
beos, lo incluye entre los manuscritos no colacionados, bien por ser apógrafos de otros manuscritos conservados, o por coincidir con manuscritos más antiguos en tal grado que apenas tienen nada nuevo que ofrecer ${ }^{4}$. Tampoco lo colaciona R. Hanhart en su edición de 1 Esdras, por incluirlo entre los manuscritos que dependen directamente de otros, pero añade, después de mencionarlo, la siguiente observación: «Die Hs. ist laut Mitteilung der Bibliothek vom 24.10.1969 im spanischen Bürgerkrieg verbrannt» (el manuscrito, según comunicación de la biblioteca de 24.10.1969, se quemó en la guerra civil española) ${ }^{5}$. En las sucesivas ediciones de otros libros en la serie de Gotinga, cuando se lo menciona, siempre aparece con una nota de remisión a esta noticia de 1 Esdras que acabo de citar, subscrita por Hanhart ${ }^{6}$.

La destrución de este manuscrito en la guerra civii española es una noticia que se repite una y otra vez en las publicaciones relativas al texto griego de la Políglota de Alcalá, la última de ellas en un artículo de A. Schenker publicado en $1994^{7}$, si bien este profesor introduce un signo de interrogación: «im Bürgerkrieg 1936-1939 verbrannt?» (¿quemado en la guerra civil de 1936-1939?).

Mi interés por el manuscrito complutense fue paralelo al de la primera noticia de Hanhart y mi formación académica en la Universidad Complutense de Madrid. En carta del Director de la Biblioteca de la Universidad de 5 de julio de 1973 proponía éste la restauración de cuatro Biblias «que sufrieron graves daños en la pasada guerra civil», una hebrea, dos latinas y la Biblia griega en cuestión: Códice $n^{\circ} 22$, Biblia griega, 307 hojas en vitela. Quemada. La propuesta es

${ }^{4}$ W. KAPPLER, Maccabaeorum liber I (Gotinga 1936) pág. 11.

5 R. HANHART, Septuaginta. VIII/l Esdrae Liber I (Gotinga 1974) pág. 14.

${ }^{6}$ El ms. 442 es supuestamente una copia del 68, y este es directamente dependiente del 731 (= Venecia, Bib. Marc. Gr. 16), cf. R. HANHART, Septuaginta. VIII/4 Iudith (Gotinga 1979) pág. 12, y R. HANHART, Septuaginta. VIII/2 Esdrae Liber II (Gotinga 1993) pág. 11: «zu der verlorengegangenen Hs. 442 vgl. Esdr I Einl. S. 14» (para el manuscrito perdido 442, cf. Esdras I, Introducción, pág. 14).

7 A. SCHENKER, «Der alttestamentliche Text in den vier grossen Polyglottenbibeln nach dem heutigen Stand der Forschung», Theologische Review 90 (1994) págs. 178188: pág. 181. 
aceptada por el Presidente de la Comisión Gestora y Técnica de la Biblioteca de la Universidad Complutense en carta del 29 de julio del mismo año ${ }^{8}$.

Pasaron tres décadas hasta que a comienzos de 2004 volví a interesarme por el estado de la restauración de este manuscrito y una posible consulta del mismo. Se me informó de que la restauración era muy lenta y difícil, que se conservaban fragmentos muy pequeños, y que sólo habían conseguido restaurarlo parcialmente. No obstante, se podía consultar la copia digitalizada de los fragmentos restaurados. Más tarde, gracias a la amabilidad de D. ${ }^{a}$ Pilar Moreno, Directora de la Unidad de Acceso al documento y digitalización de la Biblioteca, pude consultar también la caja que contiene todos los fragmentos que se conservan de este códice ${ }^{9}$.

Este breve artículo tiene como finalidad informar ante todo de la existencia del códice, de su estado de conservación y del contenido actual del mismo. En primer lugar, por tratarse de un códice griego utilizado por los colaboradores de la Políglota de Alcalá que se creía destruido en la guerra civil española. En segundo lugar, porque he podido comprobar que se conserva más de lo que cabía esperar a partir de los anteriores informes sobre su incendio, a saber, libros enteros o casi enteros y fragmentos de desigual tamaño de otros libros. Y además, porque tal vez no carezca de interés para futuras ediciones de la Septuaginta en la serie de Gotinga, tanto de los libros históricos como de los sapienciales. En efecto, aunque el manuscrito complutense sea copia del 68, a su vez copia del 731 (Venecia, Bibl. Marc. $G r$ 16, s. XIV) ${ }^{10}$, este último en algunos libros es muy defec-

\footnotetext{
${ }^{8}$ Un informe del Profesor José Simón Díaz, dirigido al Vice-Rector de dicha Universidad con fecha del 11 de septiembre de 1973, advierte de que «conviene saber los perjuicios que pueden derivarse de impedir el uso de los mismos [códices] durante un largo período, que en un caso anterior ha superado los diez años, ya que tengo noticia de que la 'Biblia hebrea' es indispensable para los trabajos que tiene en curso el departamento correspondiente».

9 Quiero agradecer al personal de la Biblioteca Histórica «Marqués de Valdecilla», y en particular a $\mathrm{D}^{\mathrm{a}}$ Pilar Moreno, todas las facilidades que me han ofrecido para la consulta del original.

${ }^{10}$ R. HANHART, Septuaginta. VIII/4 Iudith (Gotinga 1979) pág. 12.
} 
tuoso: «Schlecht erhalten und oft unlesbar» (mal conservado y con frecuencia ilegible), se dice de su texto en 2 y 3 Macabeos ${ }^{11}$. En otros libros puede contribuir a definir el grupo de manuscritos al que pertenece, ayudar a esclarecer la lectura de otros pasajes, y aportar algunas lecturas nuevas, puesto que no hay una copia totalmente idéntica con el apógrafo. Y sobre todo, un estudio más detenido permitirá determinar en qué medida fue utilizado y seguido por los filólogos complutenses para la columna griega de los distintos libros.

\section{MATERIAL CONSERVADO DEL MANUSCRITO 442}

Según el catálogo de Rahlfs ${ }^{12}$, la copia de Madrid contenía los siguientes libros bíblicos y por este orden: Jueces, Rut, 1-4 Reyes, 1-2 Paralipómenos, Proverbios, Eclesiastés, Cantar de los Cantares, 1-2 Esdras, Ester (la parte que existe en hebreo), Sabiduría, Judit, Tobit y 1-3 Macabeos. En el material conservado, siguiendo el orden de Rahlfs, incluyo tanto lo que ya está restaurado y digitalizado como las partes que no están restauradas.

Material restaurado y digitalizado ${ }^{13}$

Jueces (según el texto A): 8,21-9,18; 21,24-25.

Rut: 1,4-6.12-14.19-21.

1 Reyes: fragmentos extensos de todo el libro desde 10,11 a 31,13 (falta el cap. 12). Las hojas están dañadas en la parte superior y en los márgenes de la página, por lo que estos fragmentos tienen numerosas lagunas.

${ }^{11}$ R. HANHART, Septuaginta. IX/2 Maccabaeorum Liber II (Gotinga 1959) pág. 8, e ID., Septuaginta. IX/3 Maccabaeorum Liber III (Gotinga 1960) pág. 8.

${ }^{12}$ A. RAHLFs, Verzeichnis, pág. 124.

${ }^{13}$ Para una descripción detallada de los dos CD-Roms, cf. más abajo el apartado 3 : Identificación de los fragmentos digitalizados. 
2 Reyes: Título; fragmentos de 1,1-2,15; 16,14-18,20; 18,24-20,2; 20,4-23,17.

3 Reyes: 1,24-47 (presumiblemente se prolonga con otros dos fragmentos ilegibles).

4 Reyes: 20,16-21,13; 23,6-21.

\section{Material ordenado pero no restaurado}

1 Crónicas: Fragmentos muy deteriorados, pero que pertenecen a los capítulos finales de 1 Crónicas. He logrado leer $\kappa \alpha \imath \imath \omega \alpha \beta \alpha \rho \chi$ -

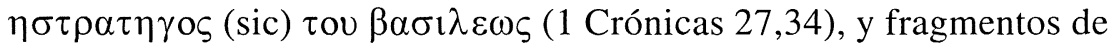
1 Crónicas 28,12-16.

2 Crónicas: Fragmentos de 2 Crónicas 1,1-13,13. Casi íntegro desde el capítulo 13,13 hasta el final del libro. Tan sólo algo dañado al principio en los márgenes internos superiores.

Proverbios: Se conserva entero con el título en tinta roja y con mayúsculas, y en el orden de capítulos de la edición de Rahlfs ${ }^{14}$.

Eclesiastés: Se conserva entero con el título en letras rojas mayúsculas. Apenas dañado en los márgenes internos superiores.

Cantar: Se conserva entero con el título en letras rojas mayúsculas y la subscripción, también con tinta roja $\tau \varepsilon \lambda$ $\varsigma_{\zeta} \tau o v \alpha \sigma \mu \alpha \tau o \zeta \tau \omega \nu$ $\alpha \sigma \mu \alpha \tau \omega \nu$. Pequeños daños en los márgenes internos superiores.

1-2 Esdras: Se conservan casi enteros con sus títulos en mayúscula y tinta roja. Algún daño en los márgenes internos superiores. El texto va precedido de un índice temático en el que se enumera el contenido de las distintas secciones.

Ester: Se conserva casi entero según el texto o' de Septuaginta y con todos los suplementos griegos ${ }^{15}$. Algo dañado en los márgenes internos superiores. El texto va precedido de un índice temático de las secciones de Ester.

\footnotetext{
${ }^{14}$ A. RAHLFS, Septuaginta, I y II (Stuttgart 1935).

${ }^{15}$ No sólo «la partie qui existe dans l'hébreu», como se indica en el catálogo de RAHLFS, Verzeichnis, pág. 124. En cambio, no tiene el añadido de Ester 5,1-2 atestiguado por la Complutense y que sigue estrechamente al texto hebreo (cf. R. HanharT, Septuaginta. VIII/3 Esther, pág. 42).
} 
Sabiduría: Se conserva casi entero, aunque algo más dañado en los márgenes internos superiores.

Judit: Se conserva casi entero, aunque va aumentando el daño en los márgenes internos superiores.

Tobit: Se conserva casi entero según el texto de los mss. BA de la

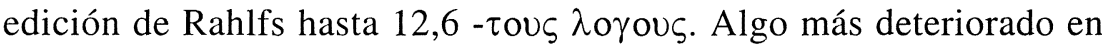
los márgenes internos superiores, excepto al final del libro.

\section{Material restaurado y digitalizado}

Tobit: Fragmentos extensos, correspondientes a la parte inferior de la página (la parte superior está quemada), desde 12,12 hasta 14,14 según el texto de los mss. BA de la edición de Rahlfs.

1 Macabeos: Fragmentos correspondientes a la parte inferior de la página desde 1,1 (sin el título) hasta 6,17.

2 Macabeos: fragmentos correspondientes a la parte inferior de la página desde 4,33 hasta 13,4.

3 Macabeos: Fragmentos desde 1,24 hasta 6,18.

\section{Observaciones}

Por esta descripción se puede deducir que el fuego afectó sobre todo a las partes iniciales y finales del manuscrito, así como al lomo del mismo y los márgenes interiores de los distintos cuadernos. En cambio, en las partes centrales del códice el texto se ha conservado casi íntegro.

Es posible que entre los fragmentos no restaurados se conserve más material de 1-4 Reyes, 1 Crónicas y 1-3 Macabeos. La carpeta que contiene los restos de las primeras hojas quemadas apenas conserva alguna palabra legible, y éstas parecen ser de 1 Reyes, no de Jueces.

No se conserva nada de la encuadernación, sólo hojas sueltas, ni se leen los números de la paginación original (probablemente escritos en los márgenes superiores quemados). 
El manuscrito, un pergamino de 315 folios, está escrito a una sola columna de 30/32 líneas por página con amplios márgenes.

\section{IDENTIFICACIÓN DE LOS FRAGMENTOS DIGITALIZADOS}

Los fragmentos restaurados y digitalizados se pueden consultar en dos CD-Roms y a través de la Biblioteca Digital Dioscórides de la Universidad Complutense ${ }^{16}$. He identificado la mayoría de esos fragmentos y me ha parecido oportuno incluir a continuación el contenido de esas identificaciones para facilitar el uso de los dos CD-Roms.

Como se podrá observar, los fragmentos digitalizados no siguen el orden original del manuscrito.

Biblia (en griego), s. XV ( = CD 1)

Consta de 30 archivos, recto y verso ${ }^{17}$.

\section{Contenido:}

1r: 3 Mac 3,29-4,5.

1v: 3 Mac 4,11-17.

2r: consta de dos fragmentos: el primero con muy pocas palabras; el segundo contiene 3 Mac 5,42-47.

$2 \mathrm{v}$ : dos fragmentos: el primero apenas tiene alguna palabra legible; el segundo contiene 3 Mac 5,5-10.

3r: dos fragmentos: el primero con alguna palabra; el segundo contiene 3 Mac 5,5-13 (bastante dañado por el margen interno).

$3 \mathrm{v}$ : dos fragmentos: el primero con alguna palabra a lo largo de cuatro líneas; el segundo contiene 3 Mac 5,23-30.

4r: Jueces 8,21-9,2 según el texto A de la edición de Rahlfs (muy dañado por los bordes).

\footnotetext{
${ }^{16} \mathrm{http} / / / \mathrm{www} . \mathrm{ucm} . \mathrm{es} / \mathrm{BUCM} / \mathrm{diosc} / \mathrm{foa} / 00 . \mathrm{htm}$.
}

${ }^{17}$ En mi copia del CD 1 falta el archivo 26v. 
4v: Jueces 9,2-18.

5r: 4 Re 20,16-20

5v: 4 Re 21,9-13.

6r: 4 Re 23,6-9.

6v: $4 \operatorname{Re} 23,17-21$.

7r: dos fragmentos: el primero contiene 3 Mac 1,24-26; el segundo, 3 Mac 1,28-2,7.

$7 \mathrm{v}$ : dos fragmentos: el primero contiene 3 Mac 3,11-15; el segundo, 3 Mac 3,16-22.

8r: 1 Mac 1,32-41 (parte inferior de la página).

8v: 1 Mac 1,57-2,3 (idem).

9r: 1 Mac 2,19-26 (idem).

9v: 1 Mac 2,40-46 (idem).

10r: 1 Mac 2,65-3,2.

10v: 1 Mac 3,16-23.

11r: 1 Mac 3,35-39.

11v: 1 Mac 3,48-58.

12r: 1 Mac 4,12-20.

12v: 1 Mac 4,34-39.

13r: 1 Mac 4,54-59.

13v: 1 Mac 5,10-15.

14r: dos fragmentos: el más pequeño con pocas palabras legibles; el segundo contiene 2 Mac 7,16-24.

14v: 2 Mac 7,33-8,1.

15r: dos fragmentos: el primero se inicia en 8,1- y contiene palabras incompletas a lo largo de 5 líneas; el segundo contiene 2 Mac 8,9-16.

15v: 2 Mac 8,23-30 (parte inferior de la página).

16r: dos fragmentos: el primero con escasas palabras de 2 Mac 10,1-3; el segundo contiene 2 Mac 9,1-7. 
16v: 2 Mac 9,15-22 (parte inferior de la página).

17r: dos fragmentos: el primero es el mismo de 16r, primero. Debe ser trasladado aquí porque continúa y complementa el fragmento segundo que contiene 2 Mac 10,2-8.

$17 \mathrm{v}$ : dos fragmentos: el primero contiene 2 Mac 10,16-18; el segundo, 2 Mac 10,17-24 (estos dos fragmentos que están separados deben ajustarse. Forman parte de un único fragmento, puesto que se solapan y completan).

18r: 2 Mac 4,33-38.

18v: 2 Mac 4,47-5,5.

19r: 2 Mac 5,12-18.

19v: 2 Mac 5,26-6,7.

20r: 2 Mac 6,19-22.

20v: 2 Mac 6,30-7,6.

21r: 2 Mac 10,32-11,2.

21v: 2 Mac 11,11-19.

22r: 2 Mac 11,28-12,3.

22v: 2 Mac 12,9-17.

23r: 2 Mac 12,22-34.

23v: 2 Mac 12,39-13,4.

24r: dos fragmentos: el primero contiene Jueces 21,24-25 según el texto A de la edición de Rahlfs; el segundo contiene Rut 1,4-6.

$24 \mathrm{v}$ : dos fragmentos: el primero contiene Rut 1,12-14; el segundo, Rut 1,19-21.

25r: 1 Re 10,11-24 (con lagunas en el medio).

25v: 1 Re 11,1-10 (con lagunas en el medio. Letra inicial del capítulo en mayúscula y con adornos).

26r: $1 \operatorname{Re} 11,15-12,9$ (con muchas lagunas).

26v: Falta en el CD 1.

27r: 1 Re 13,5-15 (con una laguna grande en el medio). 
27v: $1 \operatorname{Re} 13,19-14,7$ (idem).

28r: 1 Re 14,12-22 (con lagunas).

28v: 1 Re 14,26-36 (quemado por el medio hasta dividirlo en dos piezas).

29r: 1 Re 14,41-51.

29v: $1 \operatorname{Re} 15,3-13$.

30r: 1 Re 15,19-31.

30v: 1 Re 16,1-13 (con gran laguna en el medio).

CD 2 (archivos 31r-58v)

\section{Contenido:}

31r: 1 Re 16,18-17,8.

31v: 1 Re 17,13-25 según el texto OL de la edición de Rahlfs (aparato crítico), con lagunas.

32r: 1 Re 17,29-34.

32v: $1 \operatorname{Re} 17,44-49$.

33r: 1 Re 18,2-7.

$33 \mathrm{v}$ : $1 \operatorname{Re} 18,21-26$ (parte inferior ilegible).

34r: 1 Re 19,8-12.

34v: $1 \operatorname{Re} 19,24-20,4$.

35r: 1 Re 20,16-25.

35v: $1 \operatorname{Re} 20,35-41$.

36r: 1 Re 21,9-13.

36v: $1 \operatorname{Re} 22,6-11$.

37r: 1 Re 22,19-23,3.

37v: $1 \operatorname{Re} 23,13-19$.

38r: 1 Re 24,3-8.

38v: $1 \operatorname{Re} 24,19-25,2$. 
39r: $1 \operatorname{Re} 25,13-19$.

39v: $1 \operatorname{Re} 25,29-35$.

40r: 1 Re 26,3-8.

40v: $1 \operatorname{Re} 26,18-24$.

41r: 1 Re 27,10-28,2.

41v: $1 \operatorname{Re} 28,14-20$.

42r: 1 Re 29,6-10.

42v: $1 \operatorname{Re} 30,11-15$.

43r: $1 \operatorname{Re} 30,24-31,2$.

43v: 1 Re 31,11-13 (apenas legible). A continuación, en mitad de

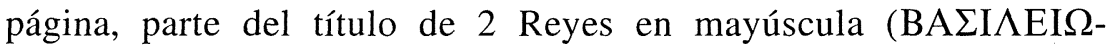
$\mathrm{N} \Delta \mathrm{E} \Upsilon \mathrm{T}[$ ), seguido de 2 Re 1,1-2.

44r: 2 Re 1,19-23.

44v: $2 \operatorname{Re} 2,10-15$.

45r: dos fragmentos: el primero contiene 2 Re 18,24-28; el segundo, 2 Re 19,1-5.

$45 \mathrm{v}$ : dos fragmentos: el primero contiene 2 Re 19,6-9; el segundo, 2 Re 19,13-18.

46r: dos fragmentos: el primero contiene 2 Re 19,19-23; el segundo, 2 Re 19,28-32.

46v: dos fragmentos: el primero contiene 2 Re 19,34-38; el segundo, 2 Re 19,42-20,2.

47r: dos fragmentos: el primero contiene 2 Re 16,14-19; el segundo, 2 Re 17,2-5.

47v: dos fragmentos: el primero contiene 2 Re 17,9-12; el segundo, 2 Re 17,17-19.

48r: dos fragmentos: el primero contiene 2 Re 17,23-27; el segundo, 2 Re 18,3-5.

48v: dos fragmentos: el primero contiene 2 Re 18,9-12; el segundo, $2 \operatorname{Re} 18,18-20$.

49r: 2 Re 20,4-17 (fragmento alargado, muy dañado por el lado izquierdo). 
49v: $2 \operatorname{Re}$ 20,10-21,6 (fragmento alargado, muy dañado por el lado derecho).

50r: 2 Re 21,1-23 (dañado por los lados y el medio).

50v: $2 \operatorname{Re} 22,1-23$ (muy dañado).

51r: 2 Re 22,29-23,2 (muy dañado, de difícil lectura).

51v: $2 \operatorname{Re} 22,5$ ?-23,2? (muy dañado e ilegible); $2 \operatorname{Re} 23,3-17$ (parte legible).

52r: 3 Re 1,24-30 (bastante dañado).

$52 \mathrm{v}: 3 \operatorname{Re} 1,40-47$.

53r: dos pequeños fragmentos con apenas dos palabras legibles. Presumiblemente continúa 3 Reyes.

$53 \mathrm{v}$ : dos pequeños fragmentos con palabras ilegibles. Presumiblemente continúa 3 Reyes, pues se puede leer el nombre de David abreviado $(\delta \alpha \delta)$.

54r: más de quince líneas con la parte inferior de la página entera: Tobit 12,12-13,5 según el texto de los mss. BA de la edición de Rahlfs.

54v: Tobit 13,8-14,3 según BA (la misma observación del anterior).

55r: Tobit 14,5-14 según BA (idem).

55v: 1 Mac 1,1-18 (veintiséis líneas íntegras de la parte inferior de la página. Pero no se conserva nada del título del libro de 1 Macabeos, ni del verso final de Tobit 14,15).

56r: 1 Mac 5,27-33 (parte inferior de la página).

56v: 1 Mac 5,44-48 (idem).

57r: 1 Mac 5,63-68 (idem).

57v: 1 Mac 6,12-17 (idem).

58r: dos pequeños fragmentos: el primero contiene 3 Mac 3,25-28; el segundo, 3 Mac 7,17-18.

$58 \mathrm{v}$ : dos pequeños fragmentos: el primero contiene 3 Mac 4,9-11; el segundo no contiene ninguna palabra entera legible. 


\section{CARÁCTER TEXTUAL DEL MANUSCRITO}

Para describir el carácter textual de este manuscrito se precisaría un estudio pormenorizado, libro por libro, de todo el material conservado. Pues es sabido que los manuscritos pueden cambiar de filiación textual de libro a libro, e incluso dentro del mismo libro. Sin embargo, los sondeos realizados sobre los fragmentos que se conservan confirman los resultados de Delitzsch para el libro de los Jueces, aunque refinados y contrastados con las ediciones más modernas. El ms. 442 se alinea en este libro claramente con el grupo $\mathrm{MNyb}_{2}$ de la edición de Brooke y McLean ${ }^{18}$, un grupo mixto tardío que, en ocasiones, puede conservar lecturas muy antiguas. Sin duda, con los manuscritos que más coincide, aunque nunca del todo, es con

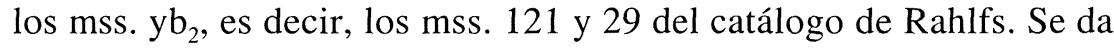
la circunstancia de que estos dos manuscritos, lo mismo que el apógrafo del 442 , el manuscrito 68 , proceden también de la Biblioteca Marciana de Venecia ${ }^{19}$. La misma imagen se obtiene para el libro de Rut a partir de los fragmentos conservados, ya que en 1,12 la singular lectura $\kappa \alpha \iota \varepsilon \gamma \varepsilon v o \mu \eta \nu \lambda \varepsilon \lambda \alpha \kappa \kappa \omega \mu \varepsilon v \eta$ está apoyada por el mismo grupo de manuscritos $\mathrm{MN}^{*} \mathrm{hyb}_{2}$.

Sondeos realizados mediante colación de algunos fragmentos conservados de Reyes (1 Re 24,3-8 y 2 Re 20,7-14) confirman que el manuscrito 422 se alinea preferentemente con el grupo de manuscritos $\mathrm{MN}$ rell o $\mathrm{MN}+$ una serie variable de minúsculos, de la edición de Brooke y McLean. Colaciones llevadas a cabo sobre fragmentos de Crónicas (2 Crónicas 13,13-18 y 36,8-23) confirman que sigue claramente al grupo dp-z de la edición de Brooke y McLean en sus lecturas específicas y cuando estos manuscritos van acompañados de la mayoría de los testigos, como puede comprobarse en la recurrente presencia de las siglas AN rell en dicha edición. Es de advertir que el ms. 442 tiene buen número de variantes exclusivas, errores gráficos,

${ }^{18}$ The Old Testament in Greek. Volume I. Part IV. Joshua, Judges and Ruth, eds. A. E. BROOKE y N. MCLEAN (Londres 1917).

${ }^{19}$ Son los mss. Gr. 3 y Gr. 2 de dicha biblioteca. Téngase en cuenta que Brooke y McLean no colacionan el ms. 68 (= Venecia, Bibl. Marc. Gr. 5), sino que sólo lo citan ocasionalmente a partir de la edición de Holmes y Parsons (1798-1827). 
a veces corregidos por otra mano en el margen o sobre la línea, y varias haplografías por homoioteleuton o homoioarcton, con frecuencia restauradas por otra mano en el margen.

Otro problema que merece un estudio aparte es en qué medida los editores complutenses se sirvieron de este manuscrito para la edición de la columna griega. Delitzsch concluye que en los libros históricos los filólogos complutenses siguieron preferentemente a los mss. 108 (= 330 de la Biblioteca Vaticana) y 442 (la copia veneciana enviada a Alcalá), pero también insiste en que no fueron éstos los únicos manuscritos utilizados por los colaboradores de la Políglota. Del Canto de Débora (Jueces 5) llega a afirmar: «Der Complutensische Septuaginta-Text des Debora-Liedes ist eine kritische Textrecension ohne irgend welche tendentiöse Textmodelung» (El texto septuagintal complutense del Canto de Débora es una recensión crítica del texto, sin ningún tipo de remodelación tendenciosa del mismo) ${ }^{20}$.

O'Connell, en un reciente estudio, llega a la conclusión de que a partir de Jueces se aprecia el peso del ms. 442 en la restauración del texto complutense: «However, with the appearance of ms. 442 for Judges, the editors had available to them a ms. which was much closer to 108. The sudden switch to 442 then becomes more understandable: in Judges the close relationship between 108 and 442, while they are not members of the same text group, offered the editors a more stable path in establishing the Greek column and provided a sense of security as to where the LXX actually lay» ${ }^{21}$.

Es una pena que los fragmentos conservados de Jueces sean tan escasos que no nos permitan contrastar esta afirmación de O'Connell por medio de colaciones más extensas. Pero pienso con Delitzsch que los complutenses siguieron preferentemente el ms. 108 de la Biblioteca Vaticana, aunque recurrieron al 442 en las lagunas de aquel

\footnotetext{
${ }^{20}$ F. DelitzsCh, Fortgesetzte Studien, pág. 17.

${ }^{21}$ S. O'Connell, How the First Edition of the Greek Old Testament was made. The Nature and Text-Critical Use of the Greek Old Testament Text of the Complutensian Polyglot Bible, Tesis doctoral, Universidad de Friburgo (Suiza) 1995, pág. 95. Agradezco al Profesor Adrian Schenker, de la Universidad de Friburgo, el haberme facilitado la consulta de esta tesis.
} 
manuscrito ${ }^{22}$, y en otras ocasiones en las que su lectura les pareció que, según sus criterios editoriales, debía preferirse. Pero en todo caso, no se debe dar la impresión de que siguieron estos dos manuscritos por igual. Predomina con mucho la fidelidad al 108, y es lo que hace que los complutenses editaran, sin saberlo, un texto luciánico o antioqueno para los libros históricos. En las colaciones realizadas sobre 2 Crónicas 36,8-23, la Complutense no sigue al ms. 442 más que esporádicamente y cuando éste coincide precisamente con el 108 , como en 2 Cr. 36,12 .

\section{RESUMEN}

El artículo informa del estado de conservación y restauración del manuscrito griego 442 = Villa-Amil 22, que fue seriamente dañado por el fuego durante la guerra civil española (1936-39). Fue uno de los manuscritos utilizados por los filólogos complutenses para la columna griega de la Políglota de Alcalá. El autor describe qué partes del manuscrito se conservan, y en qué estado se encuentran. También identifica los contenidos de los dos CD-Roms con el material restaurado y digitalizado. Finalmente, adelanta algunas observaciones sobre el carácter textual del manuscrito y su utilización en la Políglota Complutense.

Palabras Clave: Políglota Complutense, Biblia griega, manuscritos, crítica textual.

\section{SUMMARY}

The present article is a report on the state of preservation and restoration of the Greek manuscript $444=$ Villa-Amil 22 that was seriously damaged by fire during the Spanish Civil War (1936-39). The manuscript was used by the Complutensian collaborators to edit the Greek column of the Alcalá Polyglot. The author describes the parts of the manuscript that are preserved as well as their state of preservation and also identifies the full contents of the two CD-Roms with the restored and digitized material. Finally the author puts forward some remarks on the textual character of the manuscript and its use in the Complutensian Polyglot.

KEYWORDS: Alcalá polyglot, Greek Bible, manuscripts, textual criticism.

${ }_{22}^{2}$ F. DELITZSCH, Fortgesetzte Studien, págs. 25-27. 

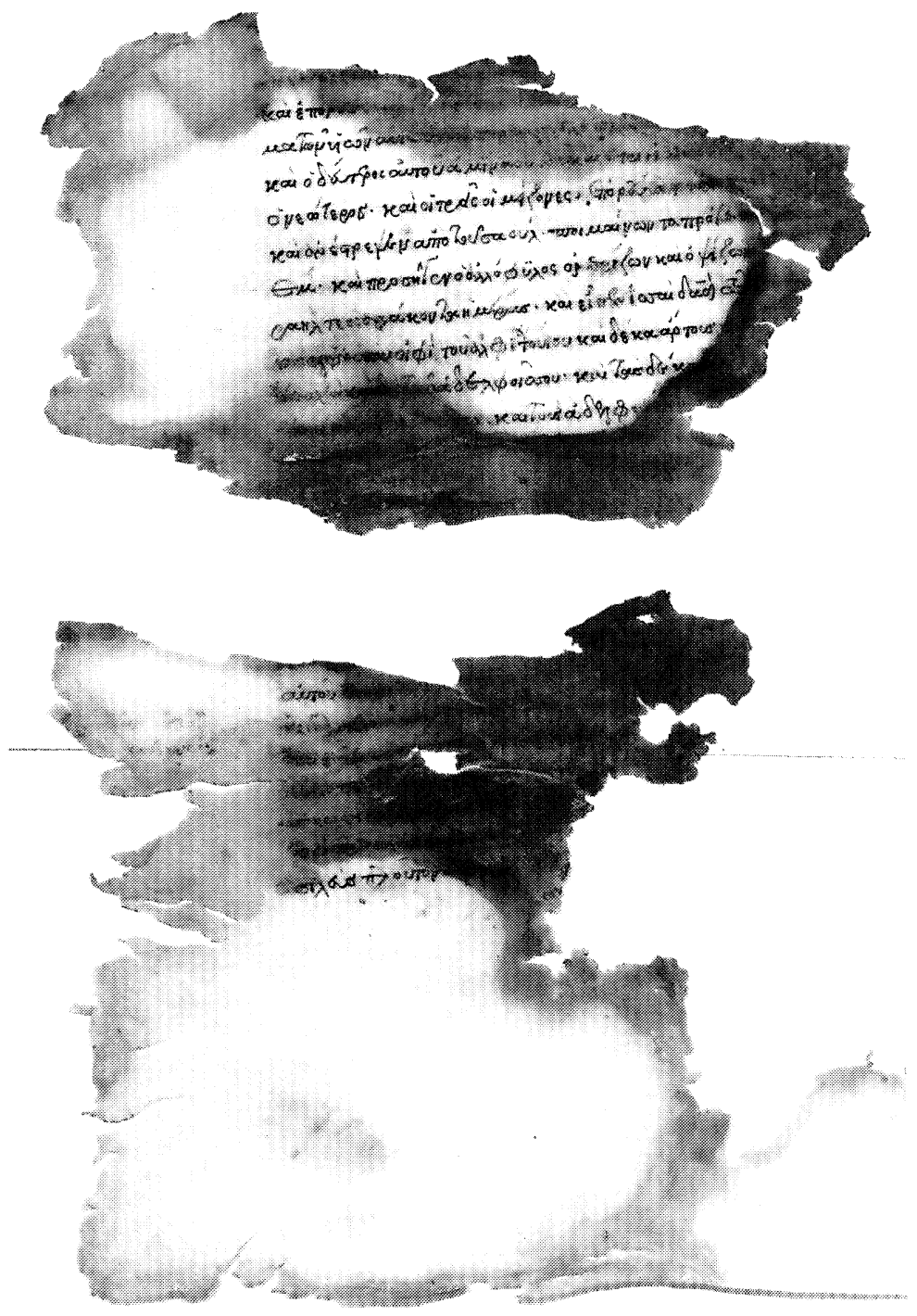

Fig. 1. CD 2, 31v: 1 Reyes 17,13-25. 

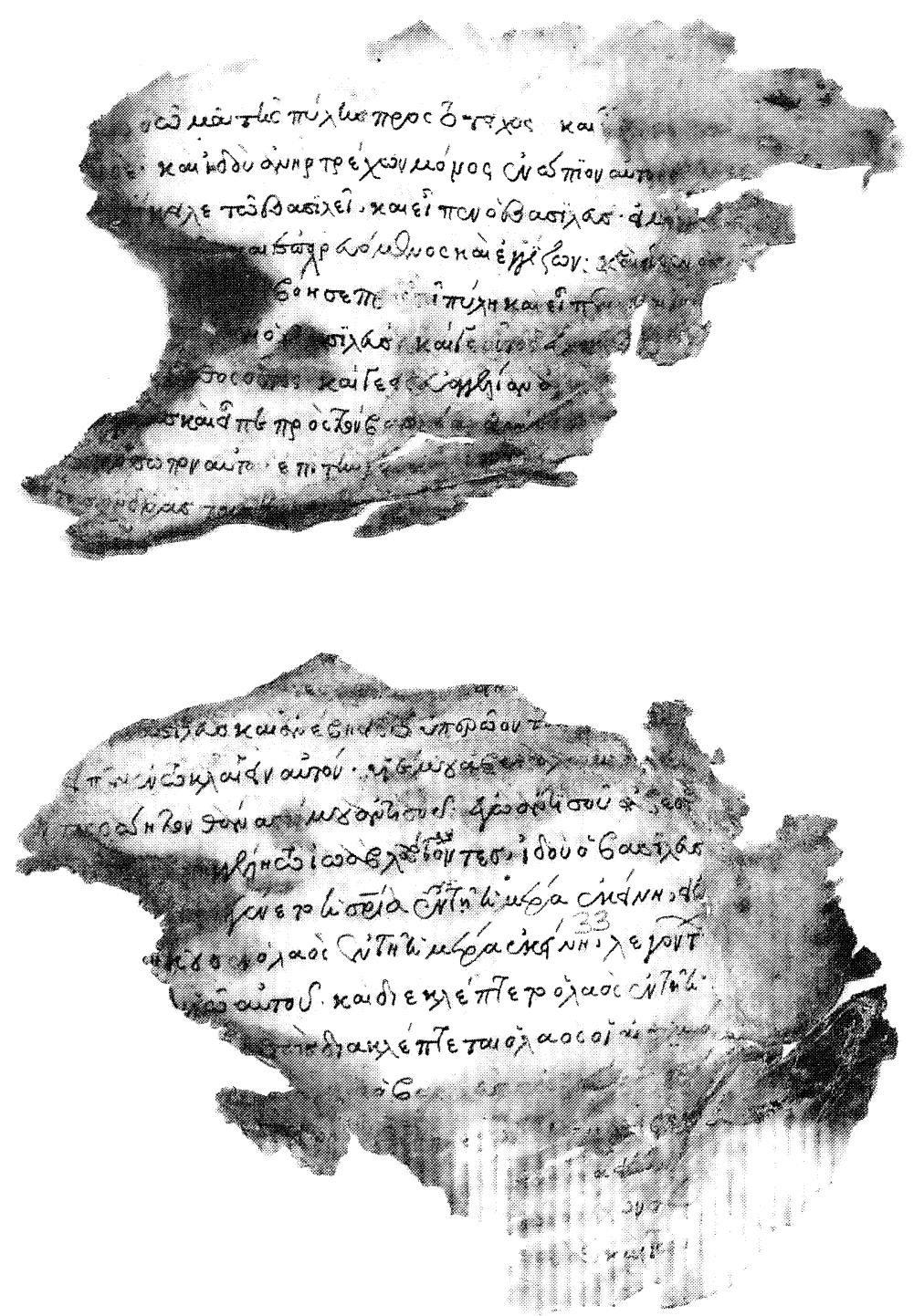

Fig. 2. CD 2, 45r: 2 Reyes 18,24-28 y 19,1-5. 


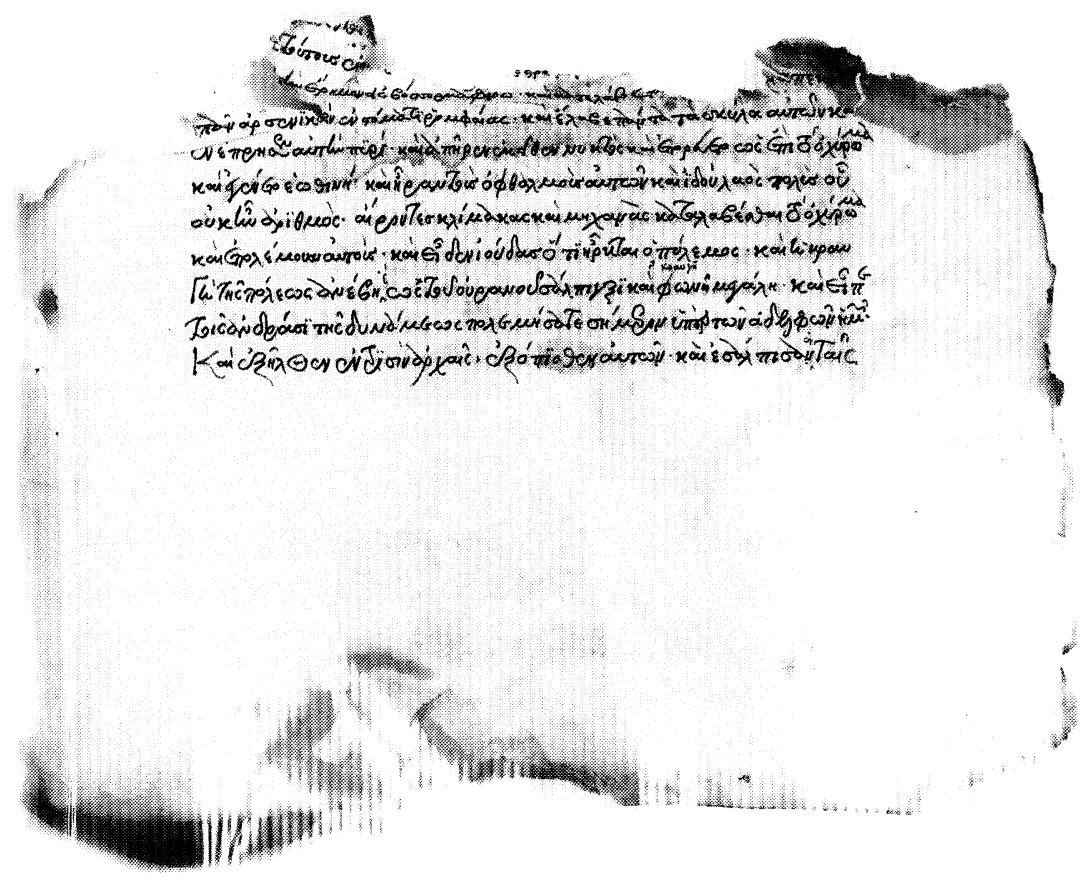

Fig. 3. CD 2, 56r: 1 Macabeos 5,27-33. 